\title{
Analysis and Applications of Backscattered Frequency Correlation Function
}

\author{
Kamal Sarabandi, Senior Member, IEEE, and Adib Nashashibi, Member, IEEE
}

\begin{abstract}
In this paper, the application of the radar backscatter frequency correlation for classification and inversion of physical parameters of terrestrial targets is investigated. Traditionally, in radar remote sensing, the backscattering coefficients and the backscatter phase difference statistics of a distributed target are considered for estimating the biophysical parameters of interest. Because of the complex nature of random media scattering problems, however, target classification and parameter inversion algorithms are very convoluted. One obvious way of enhancing the success and accuracy of an inversion algorithm is to expand the dimension of the input vector space. Depending on the radar parameters, such as footprint (pixel) size, incidence angle, and the target attributes (physical parameters), the backscatter signal decorrelates as function of frequency. In this paper, analytical and experimental procedures are developed to establish a relationship between the complex frequency correlation function (FCF) of the backscatter and the radar and target attributes. Specifically, two classes of distributed targets are considered: 1) rough surfaces and 2) random media. Analytical expressions for the frequency correlation function are derived and it is shown that the effect of radar parameters can be expressed explicitly and thus removed from the measured correlation functions. The University of Michigan wideband polarimetric scatterometer systems are used to verify the theoretical models and inversion algorithms developed in this study.
\end{abstract}

Index Terms-Frequency correlation function, radar backscatter, random media.

\section{INTRODUCTION}

W ITH the rapid industrial and human population growth since the turn of the century, the demand for accurate remote-sensing instruments for monitoring environmental changes and management of natural resources is increasing. Due to their all-weather operation capability, radar systems operating at microwave frequencies have long been proposed and implemented as powerful remote-sensing tools in retrieving the physical parameters of interest. With the advances in technology, the initial incoherent scatterometer systems evolved into high-resolution synthetic aperture radars (SAR's), fully polarimetric SAR's, and, most recently, interferometric SAR's (INSAR's). During the past three decades, considerable efforts have been devoted toward the development of scattering models and inverse scattering techniques for interpreting the

Manuscript received May 16, 1997; revised January 26, 1998. This work was supported by the Institute for Remote Sensing Applications, Joint Research Center of European Commission, Ispra, Italy.

The authors are with the Radiation Laboratory, Department of Electrical Engineering and Computer Science, The University of Michigan, Ann Arbor, MI 48109-2122 (e-mail: saraband@eecs.umich.edu).

Publisher Item Identifier S 0196-2892(99)03472-5. backscatter measurements of different terrestrial targets, such as rough surfaces and vegetation.

The electromagnetic wave interactions with scatterers in a random medium, say, a forest canopy, is a complex process that depends on the target attributes, such as size, shape, and orientation distributions, and dielectric properties of the constituent particles as well as the particle arrangement and medium architecture. For most remote-sensing applications, the gross parameters of the target, such as vertical extent, density, and total biomass, are often the parameters of interest. The electromagnetic observations, however, are often sensitive to a much larger number of target parameters. In order to obtain the parameters of interest, all of the fine features of the forest canopy must be extracted, for which accurate scattering models and reliable inversion techniques as well as a large number of radar observations are needed. Polarization and frequency diversity are often used to increase the number of independent radar observations [1]-[3]. Interferometric SAR's provide two additional independent measurements sensitive to target parameters that are also being considered for retrieval algorithms [4]. It should be noted, however, that implementation of simultaneous multifrequency, multipolarization, and interferometric SAR systems is prohibitively expensive.

In the search for an alternative and more effective approach, the application of the complex frequency correlation function (FCF) of the radar backscatter response for retrieving the physical parameters of terrestrial targets is investigated in this paper. As will be shown, in the microwave region, the decorrelation behavior of the backscatter response from a random medium is mostly dependent on the structure and distribution of the medium, with almost no dependence on the specific parameters that describe elements constituting the random medium. This is due to the fact that the scattering from a given element changes only slightly with frequency over the region where the entire target decorrelates. In other words, frequency decorrelation is due to changes in the relative positions between the scatterers (hence, changes in the relative phase angles between them) and not on the scatterers themselves. Thus, whereas correlation with respect to polarization is dependent on both the scatterers of a random medium as well as the structure of the medium, correlation with respect to frequency is dependent on target structure only, facilitating the easy extraction of the desired parameters. The literature concerning the use of frequency correlation of the radar backscatter response dates back to the original work of Ransone and Write [5], in which a two-frequency radar $(\Delta k$-radar) was used to obtain additional information about 
long ocean waves through their effects on shorter waves. Perhaps independently, Weissman [6], [7] demonstrated the application of two-frequency radars for the measurement of ocean waves heights using the Kirchhoff approximation of rough surface scattering. Another reported application of frequency correlation behavior of radar backscatter pertains to the characterization of backscatter statistics of distributed targets [8]. Using wideband radars and the backscatter at frequency steps wider than the decorrelation bandwidth of the target, the required independent samples are generated [9]. The independent samples obtained from the frequency response reduce the number of independent measurements needed to construct the statistics of the backscatter. Correlation of the scattered fields along different scattering directions have also been studied [10], with particular emphasis on its application to the reduction of radar clutter. The relationship between the angular and frequency correlation functions of a target has been established [11]. This study is of particular interest because the outcome of this investigation can directly be applied in characterizing the response of a target to an interferometric SAR.

In what follows, the complex FCF's of random media and rough surfaces are studied for the purpose of establishing a relationship between the physical parameters of the target and the measured correlation functions. Analytical expressions for the FCF are derived and compared to experimental results for different terrestrial targets. It is shown that the effect of radar parameters can be expressed explicitly and thus removed from the measured correlation function. In Section II, the FCF of the backscattered field is defined. In Section III, analytical expressions for the FCF's of the backscattered fields from both a random rough surface and a statistically homogeneous layer of random scatterers above a dielectric half-space are derived. Then, numerical simulations, demonstrating the dependence of FCF on the physical and electrical properties of random media, and controlled experiments, demonstrating the validity of the derived theoretical models, are reported in Section IV.

\section{THE FCF}

Advanced radars and scatterometers use a wideband signal as a means for measuring the range or enhancing the range resolution. The standard technique is to transmit a linear FM or stepped-frequency modulation (chirped signal) and pass the received signal through a matched filter to compress the signal to achieve the desired range resolution. In this process, the frequency response of the target is measured coherently, which can be used to calculate the complex FCF of the backscatter. Obviously, much more can be inferred from the complex FCF than the traditional $\Delta k$-radars, which can provide only the magnitude of the correlation function at a single point corresponding to a specific frequency shift.

Consider a radar system with bandwidth $B$ illuminating a distributed target. Suppose a sequence of independent measured backscattered fields from the distributed target is available at many discrete frequency points within the radar bandwidth. If the bandwidth of the radar is a small fraction of the center frequency $\left(f_{0}\right)$, it is expected that the backscat- tering coefficient be independent of frequency over the radar bandwidth, i.e.,

$$
\left\langle|E(f)|^{2}\right\rangle \simeq\left\langle\left|E\left(f_{0}\right)\right|^{2}\right\rangle .
$$

Under this assumption, the backscatter random process $E(f)$ becomes, approximately, a stationary process. Therefore, the covariance function of the process is only a function of frequency shift

$$
C\left(f_{1}, f_{2}\right)=\left\langle E\left(f_{2}\right) E^{*}\left(f_{1}\right)\right\rangle=C\left(f_{2}-f_{1}\right) .
$$

The complex FCF of this process is defined as the normalized covariance function whose magnitude and phase are given by

$$
\begin{aligned}
& R(\Delta f)=\left|\frac{C(\Delta f)}{C(0)}\right| \\
& \Phi(\Delta f)=\angle \frac{C(\Delta f)}{C(0)} .
\end{aligned}
$$

It is obvious that, when $\Delta f=0$, the FCF takes its maximum value of unity and, as $\Delta f$ increases, $R(\Delta f)$ approaches zero. In practice, the FCF is characterized approximately from $N_{s}$ independent sample measurements and $N_{f}$ frequency points. Let us denote the electric field of the $i$ th spatial sample and $j$ th frequency point by $E_{i, j}$. Then

$$
C(\Delta f)=\frac{1}{N_{s}} \sum_{i=1}^{N_{s}} \frac{1}{N_{f}} \sum_{j=1}^{N_{f}-M}\left(E_{i, j} E_{i, j+M}^{*}\right)
$$

where $\Delta f=M B / N_{f}$.

\section{THEORETICAL ANALYSIS}

In this section, analytical expressions for the backscatter FCF of rough random surfaces and a statistically homogeneous layer of random scatterers above a dielectric half-space are obtained. The expressions are explicitly expressed in terms of radar and target parameters.

\section{A. FCF for Rough Surfaces}

An analytical expression for the backscatter FCF of rough surfaces was obtained by Weissman [6], [7] using the Kirchhoff approximation for a Gaussian beam radar system. In what follows, his results are extended to a more general class of rough surfaces and for polarimetric radars with arbitrary radiation characteristics.

Consider a narrow-beam polarimetric radar system of bandwidth $B$ located at an altitude $h$ on the $z$-axis of a coordinate system, illuminating a random rough surface at an incidence angle $\theta$, as shown in Fig. 1. Let us assume that the rough surface is characterized by the height random process $z(x, y)$, which is measured with respect to the $z=0$ plane. A Gaussian probability density function (p.d.f.) is chosen for $z(x, y)$, as the height p.d.f. of most natural surfaces are found to be Gaussian [12]. The standard deviation of the height function, denoted by $s$, will be referred to as the rms height of the surface. The backscattering coefficients of a random surface are usually complex functions of the rms height, the surface correlation function, and its dielectric constant. However, in 


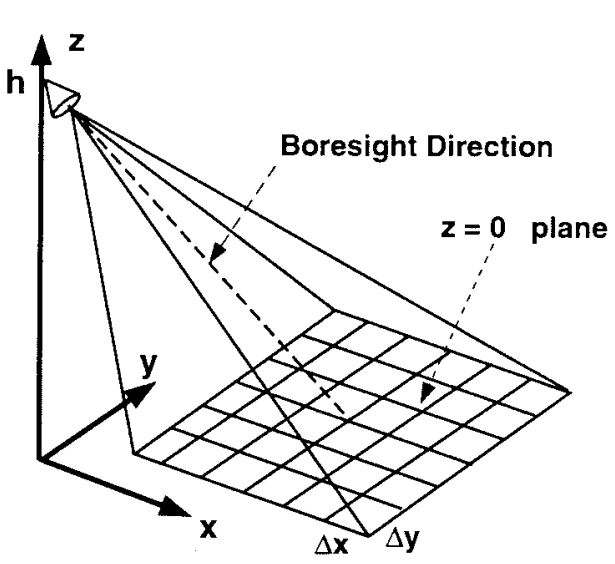

Fig. 1. Geometry of a radar system illuminating a homogeneous distributed target.

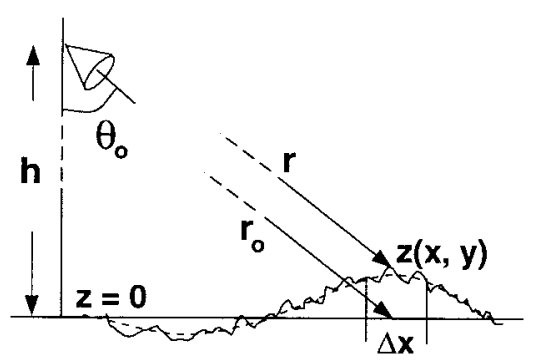

Fig. 2. Dependence of the distance $r$ on the rough surface height $z(x, y)$.

this analysis, the mathematical complexity associated with the calculation of the scattered field is circumvented by using some statistical and physical properties of the scattered field. To accomplish this, certain assumptions regarding the statistical properties of the surface must be made. Here we assume that the surface has a gentle slope as far as the large-scale surface roughness is concerned. In this case, the surface rms height is dominated by the large-scale surface roughness. Under this assumption, the scattering process becomes localized over the surface. The geometry of a typical surface sample considered in this paper is shown in Fig. 2. This class of rough surfaces include Kirchhoff surfaces considered in [6] and [7].

To derive an expression for the FCF, let us first subdivide the illuminated area (antenna footprint) into small pixels whose dimensions $\Delta x$ and $\Delta y$ are slightly larger than the scattered field correlation distance. Under this assumption, the scattering matrix associated with each pixel is a random variable that is statistically independent of the scattering matrices of the other pixels [13]. The measured scattered field $\partial \bar{E}^{s}$ associated with a pixel centered around $(x, y)$ can be obtained from

$$
\partial \bar{E}^{s}=\frac{e^{2 i k r(x, y)}}{r^{2}(x, y)} \Delta \overline{\mathbf{S}}(x, y) \bar{E}^{\text {in }}
$$

where $k$ is the free space wavenumber $(k=2 \pi / \lambda, \lambda$ is the wavelength), $\Delta \overline{\mathbf{S}}$ is the differential scattering matrix of the pixel, $\bar{E}^{i n}$ is the incident field, and $r$ is the distance from the pixel center to the radar, which is given by

$$
r(x, y)=\sqrt{x^{2}+y^{2}+(h-z)^{2}}=\sqrt{r_{o}^{2}+z^{2}-2 h z}
$$

where $r_{o}(x, y)=\sqrt{x^{2}+y^{2}+h^{2}}$. Equation (2) is valid for a distortionless radar with a uniform illumination; however, in practice, polarimetric radar measurements are corrupted by imperfections (distortion parameters) in the radar system known as systematic errors that can be removed from the measured quantities. In this case, the measured scattered field can be expressed as

$$
\partial \bar{E}^{s}=\frac{e^{2 i k r(x, y)}}{r^{2}(x, y)} \overline{\mathbf{R}}(x, y) \Delta \overline{\mathbf{S}}(x, y) \overline{\mathbf{T}}(x, y) \bar{E}^{\mathrm{in}}
$$

where $\overline{\mathbf{R}}(x, y)$ and $\overline{\mathbf{T}}(x, y)$ are the radar distortion matrices of the receiver and transmitter, respectively, and functions of the antenna radiation patterns and imbalances associated with the radar channels [13]. The total scattered field measured by the radar is the coherent addition of the scattered fields from the individual pixels, that is

$$
\begin{array}{r}
\bar{E}^{s}(f)=\left[\sum_{m} \sum_{n} \frac{e^{2 i k r\left(x_{m}, y_{n}\right)}}{r^{2}\left(x_{m}, y_{n}\right)} \overline{\mathbf{R}}\left(x_{m}, y_{n}\right)\right. \\
\left.\Delta \overline{\mathbf{S}}\left(x_{m}, y_{n}\right) \overline{\mathbf{T}}\left(x_{m}, y_{n}\right)\right] \cdot \bar{E}^{\text {in }} .
\end{array}
$$

For simplicity, let us assume that the cross-talk factors of the antenna are small, which simplifies $\overline{\mathbf{R}}$ and $\overline{\mathbf{T}}$ into diagonal matrices [13]. In this case

$$
\begin{aligned}
& E_{p q}^{s}(f) \\
& \quad=\sum_{m} \sum_{n} \frac{e^{2 i k r\left(x_{m}, y_{n}\right)}}{r^{2}\left(x_{m}, y_{n}\right)} R_{p p}\left(x_{m}, y_{n}\right) T_{q q}\left(x_{m}, y_{n}\right) \Delta S_{p q}
\end{aligned}
$$

where $p$ and $q$ denote, respectively, the transmit and receive polarizations. Without loss of generality, the amplitude of the incident field $E_{q}^{\text {in }}$ is assumed to be unity in (5). The product $R_{p p}\left(x_{m}, y_{n}\right) T_{q q}\left(x_{m}, y_{n}\right)$ is proportional to the variations in the transmit and receive antennas over the illuminated area, and henceforth will be denoted by $G_{p q}\left(x_{m}, y_{n}\right)$. This product can be obtained by calibrating the radar over its entire mainlobe, as demonstrated by Sarabandi et al. [13].

The covariance function of the measured scattered field can be computed using (5) and is given by

$$
\begin{aligned}
\left\langle E_{p q}^{s}\left(f_{2}\right) E_{p q}^{s *}\left(f_{1}\right)\right\rangle & =\sum_{m} \sum_{n} \sum_{m^{\prime}} \sum_{n^{\prime}} G_{p q}\left(f_{2}, x_{m}, y_{n}\right) G_{p q}^{*}\left(f_{1}, x_{m^{\prime}}, y_{n^{\prime}}\right) \\
& \cdot\left\langle\frac{e^{2 i\left[k_{2} r\left(x_{m}, y_{n}\right)-k_{1} r\left(x_{m^{\prime}}, y_{n^{\prime}}\right)\right]}}{r^{2}\left(x_{m}, y_{n}\right) r^{2}\left(x_{m^{\prime}}, y_{n^{\prime}}\right)} \Delta S_{p q}\left(f_{2}, x_{m}, y_{n}\right)\right. \\
& \left.\cdot \Delta S_{p q}^{*}\left(f_{1}, x_{m^{\prime}}, y_{n^{\prime}}\right)\right\rangle .
\end{aligned}
$$

If the radar system is in the far-field region of each pixel, the distance function $r$ can be approximated by $r(x, y) \simeq r_{o}(x, y)$ for the denominator term in (6) and by

$$
r(x, y) \simeq r_{o}(x, y)-\frac{h z(x, y)}{r_{o}(x, y)}
$$


for the phase term. In this case, the covariance function can be expressed as

$$
\begin{aligned}
& \left\langle E_{p q}^{s}\left(f_{2}\right) E_{p q}^{s *}\left(f_{1}\right)\right\rangle \\
& =\sum_{m} \sum_{n} \sum_{m^{\prime}} \sum_{n^{\prime}} \frac{e^{2 i\left[k_{2} r_{o}\left(x_{m}, y_{n}\right)-k_{1} r_{o}\left(x_{m^{\prime}}, y_{n^{\prime}}\right)\right]}}{r_{o}^{2}\left(x_{m}, y_{n}\right) r_{o}^{2}\left(x_{m^{\prime}}, y_{n^{\prime}}\right)} \\
& \cdot G_{p q}\left(f_{2}, x_{m}, y_{n}\right) G_{p q}^{*}\left(f_{1}, x_{m^{\prime}}, y_{n^{\prime}}\right) \\
& \cdot\left\langle\operatorname { e x p } \left(2 i h \left[ k_{1} \frac{z\left(x_{m^{\prime}}, y_{n^{\prime}}\right)}{r_{o}\left(x_{m^{\prime}}, y_{n^{\prime}}\right)}-k_{2} \frac{z\left(x_{m}, y_{n}\right)}{\left.\left.r_{o}\left(x_{m}, y_{n}\right)\right]\right)}\right.\right.\right. \\
& \left.\cdot \Delta S_{p q}\left(f_{2}, x_{m}, y_{n}\right) \Delta S_{p q}^{*}\left(f_{1}, x_{m^{\prime}}, y_{n^{\prime}}\right)\right\rangle .
\end{aligned}
$$

The expression in (7) can be simplified further if the following observations are taken into consideration.

1) Since the rms slope of the large-scale roughness is considered to be small, it is reasonable to assume that the exponential function and the scattering amplitudes are mutually uncorrelated.

2) The shift in frequency is relatively small compared to the center frequency, therefore, the backscattering amplitude does not vary significantly over $\Delta f=f_{2}-f_{1}$, that is

$$
\begin{gathered}
\left\langle\Delta S_{p q}\left(f_{2}, x_{m}, y_{n}\right) \Delta S_{p q}^{*}\left(f_{1}, x_{m}, y_{n}\right)\right\rangle \\
\simeq\left\langle\left|\Delta S_{p q}\left(f_{1}, x_{m}, y_{n}\right)\right|^{2}\right\rangle=\sigma_{p q}^{o} .
\end{gathered}
$$

Here $\sigma_{p q}^{o}$ is the radar backscattering coefficient of the random rough surface.

3) Pixel size is chosen to be larger than the scattered field correlation distance; hence, $\Delta S_{p q}\left(f_{2}, x_{m}, y_{n}\right)$ is independent of $\Delta S_{p q}\left(f_{1}, x_{m^{\prime}}, y_{n^{\prime}}\right)$ when $m \neq m^{\prime}$ and $n \neq n^{\prime}$.

Under these conditions, the ensemble average term in (7) can be expressed as follows:

$$
\begin{aligned}
\left\langle\exp \left(2 i h\left[k_{1} \frac{z\left(x_{m^{\prime}}, y_{n^{\prime}}\right)}{r_{o}\left(x_{m^{\prime}}, y_{n^{\prime}}\right)}-k_{2} \frac{z\left(x_{m}, y_{n}\right)}{r_{o}\left(x_{m}, y_{n}\right)}\right]\right)\right. \\
\left.\cdot \Delta S_{p q}\left(f_{2}, x_{m}, y_{n}\right) \Delta S_{p q}^{*}\left(f_{1}, x_{m^{\prime}}, y_{n^{\prime}}\right)\right\rangle \\
=\left\langle\exp \left(2 i h\left[k_{1} \frac{z\left(x_{m^{\prime}}, y_{n^{\prime}}\right)}{r_{o}\left(x_{m^{\prime}}, y_{n^{\prime}}\right)}-k_{2} \frac{z\left(x_{m}, y_{n}\right)}{r_{o}\left(x_{m}, y_{n}\right)}\right]\right)\right\rangle \\
\cdot \cdot\left\langle\Delta S_{p q}\left(f_{2}, x_{m}, y_{n}\right) \Delta S_{p q}^{*}\left(f_{1}, x_{m^{\prime}}, y_{n^{\prime}}\right)\right\rangle \\
= \begin{cases}C_{\mathrm{surf}}^{p q}(\Delta f) \Delta x \Delta y, & m=m^{\prime} \text { and } n=n^{\prime} \\
0, & m \neq m^{\prime} \text { and } n \neq n^{\prime}\end{cases}
\end{aligned}
$$

where $C_{\text {surf }}^{p q}(\Delta f)$ is the covariance function of the rough surface independent of the radar system and is given by

$$
C_{\text {surf }}^{p q}(\Delta f)=\left\langle\exp \left(-2 i \frac{h}{r_{o}\left(x_{m}, y_{n}\right)} z\left(x_{m}, y_{n}\right) \Delta k\right)\right\rangle \sigma_{p q}^{\circ} \text {. }
$$

Upon substituting (8) into (7) and replacing the summations with integrals, we arrive at the following expression for the measured covariance function

$$
\begin{aligned}
\left\langle E_{p q}^{s}\left(f_{2}\right) E_{p q}^{s *}\left(f_{1}\right)\right\rangle & \\
= & {\left[\iint_{e^{2 i \Delta k r_{o}(x, y)}} \frac{e^{2 i}(x, y)}{r_{o}^{4}(x, y)} G_{p q}\left(f_{2}, x, y\right) G_{p q}^{*}\left(f_{1}, x, y\right) d x d y\right] } \\
& \cdot C_{\text {surf }}^{p q}(\Delta f)
\end{aligned}
$$

where $\Delta k=2 \pi \Delta f / c$ and $c$ is the speed of light.

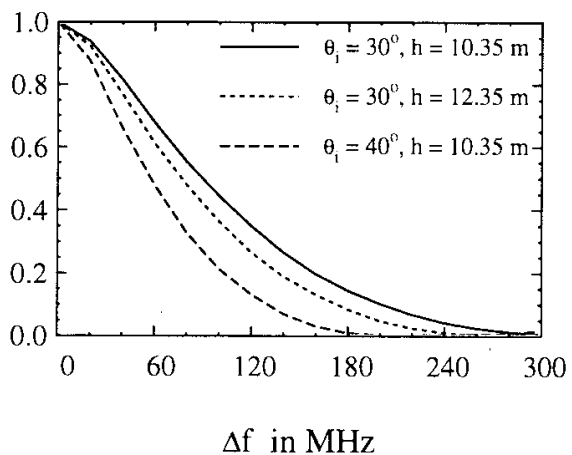

Fig. 3. System correlation function $R_{\mathrm{sys}}^{v v}(\Delta f)$ of a C-band radar $\left(5^{\circ}\right.$ beamwidth and $400 \mathrm{MHz}$ bandwidth) computed for different incidence angles and antenna heights.

Using (10), the following expression for the magnitude of FCF is obtained:

$$
R_{\mathrm{msr}}^{p q}(\Delta f)=R_{\mathrm{sys}}^{p q}(\Delta f) R_{\mathrm{surf}}^{p q}(\Delta f)
$$

where

$R_{\mathrm{sys}}^{p q}(\Delta f)=$

$$
\begin{aligned}
& \left\{\frac{\left|\iint \frac{e^{i(4 \pi / c) r_{o}(x, y) \Delta f}}{r_{o}^{4}(x, y)} G_{p q}\left(f_{2}, x, y\right) G_{p q}^{*}\left(f_{1}, x, y\right) d x d y\right|}{\iint \frac{\left|G_{p q}(x, y)\right|^{2}}{r_{o}^{4}(x, y)} d x d y}\right\} \\
& R_{\mathrm{surf}}^{p q}(\Delta f)=\left|\frac{C_{\text {surf }}^{p q}(\Delta f)}{C_{\text {surf }}^{p q}(0)}\right|
\end{aligned}
$$

It is noted from (11) that the measured FCF of a rough surface is composed of two terms, the first is $R_{\mathrm{sys}}^{p q}(\Delta f)$, which characterizes the system influence on the measured FCF, and the second term is $R_{\text {surf }}^{p q}(\Delta f)$, which is the FCF of the rough surface independent of the radar system used. This factorial decomposition is only valid for a homogeneous rough surface. Characterization of the radar-dependent term allows for the extraction of the surface-dependent term from the measured FCF, which in turn can be used to retrieve the physical parameters of the surface. The system FCF $R_{\mathrm{sys}}^{v v}(\Delta f)$ of a Cband scatterometer of $5^{\circ}$ beamwidth and $400-\mathrm{MHz}$ bandwidth is plotted in Fig. 3 for different incidence angles and antenna heights. The antenna patterns of the system, which will be used later on, were measured according to the procedure outlined by Sarabandi et al. [13]. It is noted (see Fig. 3) that for a given radar with fixed beamwidth and bandwidth, an increase in the effective antenna spot size along the range, due to an increase in either the incidence angle or antenna height, causes $R_{\mathrm{sys}}(\Delta f)$ to decrease at a higher rate. This behavior would mask the effect of the target component of FCF $\left[R_{\text {surf }}(\Delta f)\right]$ on the measured FCF $\left[R_{\mathrm{msr}}(\Delta f)\right]$.

The first term in (9) is simply the characteristic function of random variable $z(x, y)$ evaluated at $2 h \Delta k / r_{o}\left(x_{m}, y_{n}\right)$. As mentioned before, experimental data indicate that the surface height $z(x, y)$ for most natural surfaces has a Gaussian p.d.f., 
thus

$$
\begin{aligned}
& \left\langle\exp \left(i \frac{2 h \Delta k}{r_{o}\left(x_{m}, y_{n}\right)} z\right)\right\rangle \\
& \quad=\exp \left(-2\left(\frac{h \Delta k}{r_{o}\left(x_{m}, y_{n}\right)}\right)^{2} s^{2}+i\left(\frac{2 h \Delta k}{r_{o}\left(x_{m}, y_{n}\right)}\right) \bar{z}\right)
\end{aligned}
$$

where $\bar{z}$ and $s^{2}$ are the mean and variance of $z(x, y)$, respectively. The covariance function given by (9) can be written as

$$
\begin{aligned}
& C_{\mathrm{surf}}^{p q}(\Delta f)=\sigma_{p q}^{\circ} e^{\left(-2 \cos ^{2} \theta_{o} \Delta k^{2} s^{2}+i 2 \cos \theta_{o} \Delta k \bar{z}\right)} \\
& R_{\text {surf }}^{p q}(\Delta f)=e^{-2 \cos ^{2} \theta_{o} \Delta k^{2} s^{2}} \\
& \Phi_{\text {surf }}^{p q}(\Delta f)=2 \cos \theta_{o} \Delta k \bar{z}
\end{aligned}
$$

Note that in this derivation it is assumed that the antenna beamwidth is narrow enough so that $h / r_{\circ}(x, y) \simeq \cos \theta_{\circ}$. It is interesting to note that FCF is only a function of the rms height of the rough surface and is independent of the effective permittivity of the lower half-space. Accurate measurements of FCF provide a means of retrieving surface roughness, as will be demonstrated in Section IV.

Monakov et al. [8] derived the FCF of a homogeneous rough surface using Kirchhoff approximation to arrive at an improved understanding of the fading characteristics of radar backscatter from rough surfaces. In their formulation, the surface correlation function was included in the analysis and the radar spot on the ground was treated as a single pixel. The analytical FCF expression reported in [8] is similar to (13) and is included here for convenience

$$
R_{\mathrm{msr}}(\Delta f)=e^{-2 \cos ^{2} \theta_{o} \Delta k^{2} s^{2}} \cdot \operatorname{sinc}\left[2 \pi c^{-1} \sin \theta_{o} \Delta f L_{x}\right]
$$

where $L_{x}$ is across-track dimension of the radar spot on the ground. The first and second terms correspond to $R_{\text {surf }}$ and $R_{\text {sys }}$, respectively. It is observed that the normalized FCF is independent of the surface correlation function despite its inclusion in the derivation.

\section{B. A Layer of Random Scatterers Above a Ground Plane}

Now let us consider a layer of thickness $d$ consisting of a collection of scatterers randomly embedded in a homogeneous medium, as shown in Fig. 4. Suppose that a narrow beam radar, situated at a height $h$ above the layer, illuminates the random medium. The scattering volume, which can be either sparse or dense, is situated above a homogeneous ground plane with either smooth or slightly rough interface. For a dense random medium composed of discrete particles, such as sand, or coagulated/connected matrix of particles, such as snow, a cluster of particles can be considered as the scatterers whose dimensions can be characterized from the field decorrelation distance in the medium. The position, size, and orientation of the scatterers within the cluster are random variables; hence, the scattering matrix of the cluster is also a random variable. In this paper, the word cluster will be used in reference to a scattering center that could be either a collection of particles (dense random medium) or an individual particle (sparse random medium).
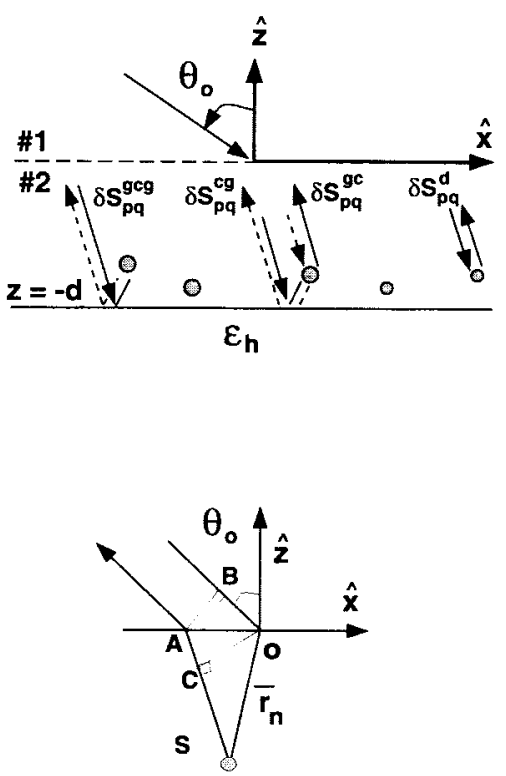

Fig. 4. Dominant scattering mechanisms contributing to the total backscatter response of a layer of random scatterers above a ground plane.

Assume that a plane wave is illuminating the random medium at an incidence angle $\theta_{o}$, as shown in Fig. 4. In addition, let the effective dielectric constant of the medium be $\epsilon_{\text {eff }}=\epsilon^{\prime}+i \epsilon^{\prime \prime}$, where $\epsilon^{\prime \prime} \ll \epsilon^{\prime}$ (low-loss medium). The incident wave at the interface between air and the random medium refracts according to Fresnel's law, in which the direction of propagation is changed from $\theta_{o}$ to $\theta_{t}$ and the magnitude of the transmitted wave is given by $E_{q}^{t}=t_{q}^{12} E_{q}^{i}$. Here $q=v$ or $h$ and $t_{q}^{12}$ is the Fresnel transmission coefficient at the interface between air and the equivalent medium. Inside the layer, a cluster is illuminated by the transmitted mean-field (a plane wave) propagating in the layer with an effective propagation constant $k_{e}\left(k_{e}=k_{o} \sqrt{\epsilon_{\mathrm{eff}}}\right)$ and a spherical scattered wave is generated. The scattered field is proportional to the scattering amplitude of the cluster in a background of $\epsilon_{\mathrm{eff}}$. In this case, the $z$-component of the propagation constant of a plane wave propagating in medium 2 is $k_{z}=\sqrt{k_{2}^{2}-k_{o}^{2} \sin ^{2} \theta_{o}}$, where $k_{2}=k_{o} \sqrt{\epsilon_{\mathrm{eff}}} \approx \beta_{2}\left(1+i \epsilon^{\prime \prime} / 2 \epsilon^{\prime}\right)$ and $\beta_{2}=k_{o} \sqrt{\epsilon^{\prime}}$. Noting that $\cos \theta_{t}$ can be approximated as $\cos \theta_{t} \approx \sqrt{1-\sin ^{2} \theta_{o} / \epsilon^{\prime}}$, it can be shown that $k_{z}$ reduces to $k_{z} \simeq \beta_{2} \cos \theta_{t}+i \alpha / \cos \theta_{t}$, where $\alpha=\beta_{2} \epsilon^{\prime \prime} / 2 \epsilon^{\prime}$. Therefore, the transmitted field at point $\bar{r}$ in medium 2 is approximated by

$$
E_{q}^{t}(\bar{r}) \approx t_{p q}^{12} e^{i\left(\bar{k}_{\perp} \cdot \bar{r}_{\perp}-k_{z} z\right)}
$$

where $\bar{k}_{\perp}=k_{x} \hat{x}+k_{y} \hat{y}$ and $\bar{r}_{\perp}=x \hat{x}+y \hat{y}$.

As mentioned before, the scattered wave is a spherical wave whose amplitude and phase variations must be accounted for. Noting that the direction of propagation of the scattered wave in the backscatter direction is given by $\hat{k}_{s}=-\sin \theta_{o} \hat{x}+$ $\cos \theta_{t} \hat{z}$ and using the origin as the reference phase center, the phase of the scattered field can be determined. Referring to Fig. 4, it can easily be shown that the phase shift due to the path length $\mathrm{OB}$ is equal to the phase shift due to the path length AC. Therefore, the extra phase shift is due to 
the path length SC only, which is given by $e^{-i \beta_{2}\left(\bar{k}_{s} \cdot \bar{r}_{n}\right)}=$ $e^{-i \beta_{2} \cos \theta_{t} z} e^{i\left(\bar{k}_{\perp} \cdot \bar{r}_{n \perp}\right)}$. In addition, as the ray propagates from $\mathrm{S}$ to $\mathrm{A}$, it also experiences attenuation that is proportional to $e^{\alpha z / \cos \theta_{t}}$. Hence, the scattered field from a scatterer located at $\bar{r}_{n}$, referenced to the origin, has the phase and magnitude coefficients $e^{i\left(\bar{k}_{\perp} \cdot \bar{r}_{n \perp}\right)} e^{-i k_{z} z}$, which is exactly the same coefficient as for the incident wave. It should be noted that for sparse medium, the interface between air and the random medium is diffuse and the incident wave will not be diffracted. The transmission coefficient in this case can be set equal to unity.

To the first order of scattering approximation, ignoring the effect of multiple scattering between the clusters, the backscatter from a cluster along the $z$-axis is composed of four components, as illustrated in Fig. 4. These components are as follows.

1) Direct backscatter from the cluster transmitted through the interface between air and the random medium. This term is denoted by $\delta E_{p q}^{d}$ and is given by

$$
\delta E_{p q}^{d}=t_{p}^{21} e^{-i k_{z} z} \Delta S_{p q}^{d} t_{q}^{12} e^{-i k_{z} z}
$$

where $\Delta S_{p q}^{d}$ is the scattering matrix of the cluster in backscatter.

2) Cluster-ground scattering term that represents the bistatic scattering contribution from the cluster reflected from the ground plane and transmitted through the interface. This term is denoted by $\delta E_{p q}^{c g}$ and is given by

$$
\delta E_{p q}^{c g}=t_{p}^{21} e^{i k_{z} d} R_{p} e^{i k_{z}(z+d)} \Delta S_{p q}^{b} t_{q}^{12} e^{-i k_{z} z} .
$$

Here $R_{p}$ is the reflectivity of the ground plane and the exponential functions account for the spherical propagation of the scattered field from the cluster to the ground plane and from the ground plane to the interface. The reflectivity term $R_{p}(p=v$ or $h)$ is simply the Fresnel reflection coefficient for smooth surfaces and for rough surfaces is the coherent reflectivity of the surface [14]. Also, $\Delta S_{p q}^{b}$ represents the $p q$ element of the differential scattering matrix in the bistatic direction, as shown in Fig. 4.

3) Ground-cluster scattering term is the reciprocal of the cluster-ground term. The transmitted wave travels to the ground plane and then is reflected from the surface. The reflected wave illuminates the cluster and the bistatic scattered field travels to the interface. This term is denoted by $\delta E_{p q}^{g c}$ and is given by

$$
\delta E_{p q}^{g c}=t_{p}^{21} e^{-i k_{z} z} \Delta S_{q p}^{b} e^{i k_{z}(z+d)} R_{q} t_{q}^{12} e^{i k_{z} d} .
$$

4) Ground-cluster-ground scattering term is generated from the backscatter of the cluster illuminated by the reflected wave, as shown in Fig. 4. This term is denoted by $\delta E_{p q}^{g c g}$ and is given by

$$
\begin{aligned}
\delta E_{p q}^{g c g}= & t_{p}^{21} e^{i k_{z} d} R_{p} e^{i k_{z}(z+d)} \Delta S_{p q}^{d d} e^{i k_{z}(z+d)} \\
& \cdot R_{q} t_{q}^{12} e^{i k_{z} d}
\end{aligned}
$$

where as before $\Delta S_{p q}^{d d}$ is the $p q$ element of the differential scattering matrix of the cluster in backscatter when illuminated by the reflected wave.
The total backscattered field from the cluster, located at point $(0,0, z)$ in the random medium, is the coherent sum of the individual components, that is

$$
\delta E_{p q}(f)=\delta E_{p q}^{d}(f)+\delta E_{p q}^{c g}(f)+\delta E_{p q}^{g c}(f)+\delta E_{p q}^{g c g}(f) .
$$

It should be emphasized that the phase reference point for the expression given by (18) is a point on the interface between air and the random medium. The scattered field from a small pixel centered at $\left(x_{m}, y_{n}\right)$ over the interface of the random medium and air (the $z=0$ plane) is the coherent sum of the scattered fields from all clusters along the transmitted ray and is given by

$$
\Delta E_{p q}\left(f, x_{m}, y_{n}\right)=\sum_{\ell} \delta E_{p q}\left(f, x_{m}, y_{n}, z_{\ell}\right) e^{i\left(k_{x} x+k_{y} y\right)}
$$

where $z_{\ell}$ denotes the height of the $\ell$ th cluster. Changing the reference phase point to be at the radar antenna and adding the contribution of all small pixels within the illuminated area, the measured scattered field can be obtained from

$$
\begin{aligned}
E_{p q}^{s}(f)= & \sum_{m} \sum_{n} \sum_{\ell} \frac{e^{2 i k r_{o}\left(x_{m}, y_{n}\right)}}{r_{o}^{2}\left(x_{m}, y_{n}\right)} \\
& \cdot G_{p q}\left(f, x_{m}, y_{n}\right) \delta E_{p q}\left(f, x_{m}, y_{n}, z_{\ell}\right) .
\end{aligned}
$$

In this case, the covariance function of the measured field can be expressed as

$$
\begin{aligned}
\left\langle E_{p q}^{s}\left(f_{2}\right)\right. & \left.E_{p q}^{s *}\left(f_{1}\right)\right\rangle \\
= & \sum_{m} \sum_{n} \sum_{\ell} \sum_{m^{\prime}} \sum_{n^{\prime}} \sum_{\ell^{\prime}} \\
& \cdot \frac{e^{2 i\left[k_{2} r_{o}\left(x_{m}, y_{n^{\prime}}\right)-k_{1} r_{o}\left(x_{m^{\prime}}, y_{n^{\prime}}\right)\right]}}{r_{o}^{2}\left(x_{m}, y_{n}\right) r_{o}^{2}\left(x_{m^{\prime}}, y_{n^{\prime}}\right)} \\
& \cdot G_{p q}\left(f_{2}, x_{m}, y_{n}\right) G_{p q}^{*}\left(f_{1}, x_{m^{\prime}}, y_{n^{\prime}}\right) \\
& \cdot\left\langle\delta E_{p q}\left(f_{2}, x_{m}, y_{n}, z_{\ell}\right) \delta E_{p q}^{*}\left(f_{1}, x_{m^{\prime}}, y_{n^{\prime}}, z_{\ell^{\prime}}\right)\right\rangle .
\end{aligned}
$$

If we assume that the cluster size is smaller than the field correlation distance, the ensemble average in (20) can be expressed as

$$
\begin{aligned}
& \left\langle\delta E_{p q}\left(f_{2}, x_{m}, y_{n}, z_{\ell}\right) \delta E_{p q}^{*}\left(f_{1}, x_{m^{\prime}}, y_{n^{\prime}}, z_{\ell^{\prime}}\right)\right\rangle \\
& \quad= \begin{cases}\left\langle\delta E_{p q}^{o}\left(f_{2}, x_{m}, y_{n}, z_{\ell}\right)\right. & \\
\left.\delta E_{p q}^{o *}\left(f_{1}, x_{m}, y_{n}, z_{\ell}\right)\right\rangle & m=m^{\prime}, n=n^{\prime}, \ell=\ell^{\prime} \\
\Delta x \Delta y \Delta z, & m \neq m^{\prime}, n \neq n^{\prime}, \ell \neq \ell^{\prime} \\
0, & \end{cases}
\end{aligned}
$$

where $\delta E_{p q}^{o}$ is the scattered field per unit volume.

Upon substituting (21) into (20) and replacing the summations with integrals, we arrive at the following expression for the measured covariance function:

$$
\begin{aligned}
\left\langle E_{p q}^{s}\left(f_{2}\right) E_{p q}^{s *}\left(f_{1}\right)\right\rangle & \\
= & {\left[\iint \frac{e^{2 i \Delta k r_{o}(x, y)}}{r_{o}^{4}(x, y)} G_{p q}\left(f_{2}, x, y\right) G_{p q}^{*}\left(f_{1}, x, y\right) d x d y\right] } \\
& \cdot C_{\mathrm{vol}}^{p q}(\Delta f)
\end{aligned}
$$

where $C_{\mathrm{vol}}^{p q}(\Delta f)$ is the covariance function for the volume scattering layer independent of the radar system

$$
C_{\mathrm{vol}}^{p q}(\Delta f)=\int\left\langle\delta E_{p q}^{o}\left(f_{2}, z\right) \delta E_{p q}^{o *}\left(f_{1}, z\right)\right\rangle d z .
$$


By normalizing the measured covariance function to its maximum value at $\Delta f=0$ and taking the absolute value, the magnitude of the FCF of the random medium can be expressed by

$$
R_{\mathrm{msr}}^{p q}(\Delta f)=R_{\mathrm{sys}}^{p q}(\Delta f) R_{\mathrm{vol}}^{p q}(\Delta f)
$$

which is similar to the expression found for the rough surface case.

The expression for the covariance function can be simplified further by invoking a few heuristic/intuitive scattering properties of random media. For a random collection of scatterers, it is expected that the ensemble average of the scattered amplitudes in both backscatter and bistatic directions be mutually uncorrelated, that is

$$
\left\langle\Delta S_{p q}^{o d} \Delta S_{p q}^{o b *}\right\rangle=0
$$

Here, $\Delta S^{\circ}$ represents scattering per unit volume $(\Delta S=$ $\left.\Delta S^{\circ} \Delta x \Delta y \Delta z\right)$. Similarly, it is also assumed that the backscattering amplitudes $\Delta S_{p q}^{d}$ and $\Delta S_{p q}^{d d}$ are uncorrelated, thus

$$
\left\langle\Delta S_{p q}^{o d} \Delta S_{p q}^{o d d *}\right\rangle=0 \text {. }
$$

Furthermore, it is assumed that the random medium is azimuthally symmetric,

$$
\left\langle\left|\Delta S_{p q}^{o d}\right|^{2}\right\rangle=\left\langle\left|\Delta S_{p q}^{o d d}\right|^{2}\right\rangle
$$

These assumptions have been demonstrated numerically, using single scattering theories, for a collection of randomly oriented flat leaves. It should be noted, however, that these assumptions may not be valid in an ordered medium where the particle size and orientation distribution functions are narrow.

Substituting (14)-(18) into (23) and taking the above assumptions into consideration, it can be shown, after some algebraic manipulations, that

$$
\begin{gathered}
C_{\mathrm{vol}}^{p q}(\Delta f)=\left|t_{p}^{21}\right|^{2}\left|t_{q}^{12}\right|^{2} \\
\cdot\left[\frac{1-e^{-\chi d}}{\chi} W_{p q p q}^{o d}\left(1+\left|R_{p}\right|^{2}\left|R_{q}\right|^{2} e^{-\chi d}\right)\right. \\
+d\left(\left|R_{p}\right|^{2} W_{p q p q}^{o b}+\left|R_{q}\right|^{2} W_{q p q p}^{o b}\right. \\
\left.\left.+2 \operatorname{Re}\left[R_{p} R_{q}^{*} W_{p q q p}^{o b}\right]\right) e^{-\chi d}\right]
\end{gathered}
$$

where $\chi=2\left(\kappa / \cos \theta_{t}-i \Delta k \sqrt{\epsilon_{\mathrm{eff}}^{\prime}} \cos \theta_{t}\right)$ and $\kappa=2 \operatorname{Im}\left[k_{e}\right]$ is the extinction coefficient in Neper per meter. Here, $W_{p q p q}^{o d}=$ $\left\langle\left|\Delta S_{p q}^{\text {od }}\right|^{2}\right\rangle$ is an element of the medium phase matrix in the backscatter direction. Similarly

$$
\begin{aligned}
& W_{p q p q}^{o b}=\left\langle\left|\Delta S_{p q}^{o b}\right|^{2}\right\rangle \\
& W_{q p q p}^{o b}=\left\langle\left|\Delta S_{q p}^{o b}\right|^{2}\right\rangle \\
& W_{p q q p}^{o b}=\left\langle\Delta S_{p q}^{o b} \Delta S_{q p}^{o b *}\right\rangle
\end{aligned}
$$

represent elements of the phase matrix in the bistatic direction. In this derivation, the reciprocity theorem was enforced in relation to the bistatic scattering amplitudes, namely, $\Delta S_{p q}^{o b}\left(\theta_{1}, \theta_{2}\right)=\Delta S_{q p}^{o b}\left(\theta_{2}, \theta_{1}\right)$.
The covariance function as expressed in (24) further simplifies for the copolarized response (i.e., $p=q$ )

$$
\begin{gathered}
C_{\mathrm{vol}}^{p p}(\Delta f)=\left|t_{p}^{21}\right|^{2}\left|t_{q}^{12}\right|^{2}\left[\frac{1-e^{-\chi d}}{\chi} W_{p p p p}^{o d}\left(1+\left|R_{p}\right|^{4} e^{-\chi d}\right)\right. \\
\left.+4 d\left|R_{p}\right|^{2} W_{p p p p}^{o b} e^{-\chi d}\right] .
\end{gathered}
$$

In this case, the covariance function $C_{\mathrm{vol}}^{p p}(\Delta f)$ is a function of the extinction coefficient and the copolarized elements of the direct and bistatic scattering phase matrices. Since the FCF is a normalized function with a maximum of unity at $\Delta f=0$, the absolute values of the bistatic and backscatter terms in (25) are dropped and only their ratio remains in the FCF expression. Normalizing (25) by $\left(\left|t_{p}^{21}\right|^{2}\left|t_{q}^{12}\right|^{2} W_{p p p p}^{o d}\right)$, the normalized copolarized covariance function can be expressed as

$$
c_{\mathrm{vol}}^{p p}(\Delta f)=\frac{1-e^{-\chi d}}{\chi}\left(1+\left|R_{p}\right|^{4} e^{-\chi d}\right)+4 d\left|R_{p}\right|^{2} Q_{p p} e^{-\chi d}
$$

where $Q_{p p}$ is the ratio between the bistatic and backscatter phase matrix copolarized elements $\left(W_{p p p p}^{o b} / W_{p p p p}^{o d}\right)$. As observed from (26), the copolarized covariance is a function of only two statistical parameters describing wave propagation and scattering in the random medium, namely, $\kappa$ and $Q_{p p}$.

As a special case, let us consider a semi-infinite medium or equivalently a medium for which $\left(\kappa d / \cos \theta_{t}\right) \gg 1$. In this case, the bistatic term in (24), which characterizes the bistatic scattering contribution of the clusters as it reflects off of the layer's lower interface, no longer contributes to the covariance function. Hence, the expression for the covariance function reduces to

$$
C_{\mathrm{vol}}^{p q}(\Delta f)=\left|t_{p}^{21} t_{q}^{12}\right|^{2} \frac{W_{p q p q}^{o d}}{\chi}
$$

and the magnitude and phase of the FCF of the semi-infinite medium are given by

$$
\begin{aligned}
& R_{\mathrm{vol}}(\Delta f)=\frac{\kappa}{\sqrt{\kappa^{2}+\left(\Delta k \operatorname{Re}\left[\sqrt{\epsilon_{\mathrm{eff}}}\right]\right)^{2}}}, \\
& \Phi_{\mathrm{vol}}(\Delta f)=-\tan ^{-1}\left(\frac{\Delta k \sqrt{\epsilon_{\mathrm{eff}}} \cos ^{2} \theta_{t}}{2 \kappa}\right) .
\end{aligned}
$$

It is noted that the FCF of a semi-infinite medium is only a function of the real part of the layer's effective index of refraction (i.e., $\operatorname{Re}\left[\sqrt{\epsilon_{\mathrm{eff}}}\right]$ ) and the extinction coefficient of the medium. In this case, $R_{\mathrm{vol}}(\Delta f)$ can be used to directly estimate the extinction coefficient.

If a slightly rough surface is present underneath the scattering volume, the effect of the backscatter from the ground plane can be added noting that the surface scattering process is independent of the volume scattering process. In this case, the total covariance function of the distributed target is given by

$$
C_{\mathrm{trgt}}^{p q}(\Delta f)=C_{\mathrm{vol}}^{p q}(\Delta f)+e^{-\chi d} C_{\mathrm{surf}}^{p q}(\Delta f)
$$

where $C_{\mathrm{surf}}^{p q}(\Delta f)$ is the covariance function of the rough surface, as given in (13). 


\section{NUMERICAL SiMULATION AND \\ EXPERIMENTAL VERIFICATIONS}

Through numerical simulations, a sensitivity analysis is performed to demonstrate the dependence of the FCF on the physical and electrical properties of random media and rough surfaces. Also, the validity of the theoretical models is demonstrated by conducting controlled experiments using wideband scatterometers.

\section{A. Sensitivity Analysis}

In the previous section, expressions for the FCF of random surfaces and volumes were derived. Specifically, it was shown that FCF can be expressed in terms of the extinction coefficient, elements of the medium phase matrix, depth, and the reflectivity of the lower half-space, as well as the radar parameters, such as polarization, incidence angle, and radiation pattern. The objective of this section is to study the behavior of the FCF to demonstrate the potential of the FCF as a parameter for inversion algorithms. Consider a tenuous random medium composed of discrete scatterers with a fractional volume less than 0.01. The medium extinction matrix can be evaluated using the optical theorem. In this case, both the extinction and the phase matrix of the medium can be computed easily. Assuming azimuthal symmetry for the orientation distribution, it can be shown that, in the forward scattering direction $\left(\theta_{s}=\theta_{i}, \phi_{s}=\phi_{i}\right)\left\langle\Delta S_{p q}(\theta, \phi ; \theta, \phi)\right\rangle=0$, when $p \neq q$ [15], and therefore, the extinction matrix reduces to a diagonal matrix. Hence, the extinction coefficient for the $p$-polarized field is given by

$$
\kappa_{p}=\frac{4 \pi n_{o}}{k} \operatorname{Im}\left[\left\langle\Delta S_{p p}(\theta, \phi ; \theta, \phi)\right\rangle\right]
$$

where $n_{o}$ is the number density of scatterers per unit volume and angles $\theta$ and $\phi$ denote the elevation and azimuth angles defining the direction of propagation. The expected value of the scattering matrix elements in the forward scattering direction is characterized by computing

$$
\begin{aligned}
& \left\langle\Delta S_{p p}(\theta, \phi ; \theta, \phi)\right\rangle \\
& \quad=\int_{0}^{\infty} d r \int_{\Omega} d \omega p(r ; \omega) \Delta S_{p p}(\theta, \phi ; \theta, \phi)
\end{aligned}
$$

where $p(r ; \omega)$ is the joint distribution function of size $(r)$ and orientation angles $(\omega)$ of the scatterers. The phase matrix elements are proportional to the incoherent scattered power per unit volume and are computed from

$$
\begin{aligned}
W_{p q p q}^{o d} & =\left\langle\left|\Delta S_{p p}\left(\theta_{s}, \phi_{s} ; \theta_{i}, \phi_{i}\right)\right|^{2}\right\rangle \\
& =n_{O} \int_{0}^{\infty} d r \int_{\Omega} d \omega p(r ; \omega)\left|\Delta S_{p p}\left(\theta_{s}, \phi_{s} ; \theta_{i}, \phi_{i}\right)\right|^{2}
\end{aligned}
$$

where subscripts $s$ and $i$ denote the scattered and incident directions, respectively.

As an example, we considered a uniform layer of leaves above a smooth ground plane. Flat square leaves with surface area $15 \mathrm{~cm}^{2}$, thickness $1.33 \mathrm{~mm}$, and permittivity $\epsilon_{s}=$ $16.7+i 7.1$ were considered for this simulation. The leaf

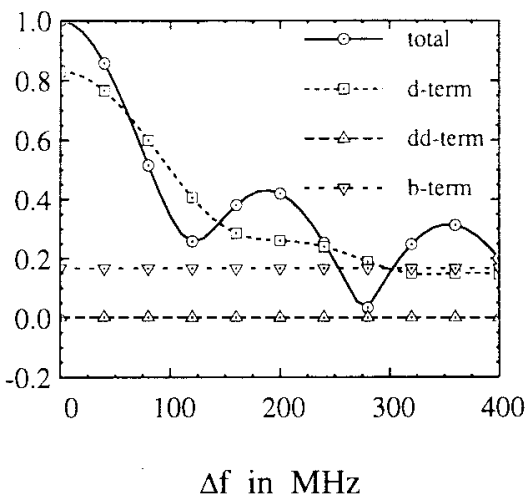

Fig. 5. Relative contribution of the direct, indirect, and bistatic terms of $R_{\mathrm{vol}}^{v v}$ of a tenuous random medium $\left(d=1 \mathrm{~m}, n_{o}=1000 / \mathrm{m}^{3} ; \theta_{o}=20^{\circ}\right)$.

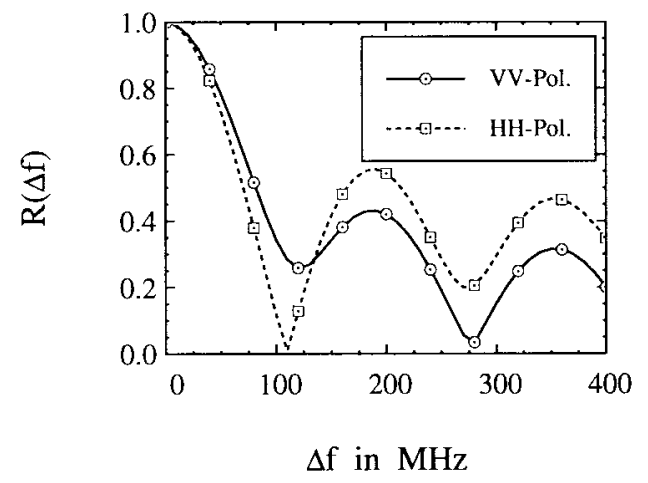

Fig. 6. Numerical simulation of the FCF of the $V V$ - and $H H$-polarized backscatter response of a tenuous random medium $\left(d=1 \mathrm{~m}, n_{o}=1000 / \mathrm{m}^{3}\right.$; $\left.\theta_{o}=20^{\circ}\right)$.

is modeled as a resistive sheet, and its scattering matrix computed following the procedure reported by Senior $e t$ al. [16]. The simulations were performed at $9.5 \mathrm{GHz}$, and a uniform probability distribution function was used for the orientation angles of the leaves. In addition, the permittivity of the lower half-space was assumed to be $\epsilon_{h}=6+i 1.5$, corresponding to a soil moisture of $m_{v}=0.15$.

To demonstrate the influence of the three major scattering mechanisms (discussed in Section III-B) on the FCF, the contribution of each scattering mechanism in (24) was computed for a random medium with $d=1 \mathrm{~m}$ and $n_{o}=1000 / \mathrm{m}^{3}$ at $\theta=20^{\circ}$ incidence angle. The FCF and the direct backscatter, indirect backscatter, and bistatic contribution to the overall FCF for the $V V$-polarized case are plotted in Fig. 5. It is shown that FCF is a strong function of the first two terms, namely, the direct backscatter and the bistatic terms but not the indirect backscatter term. This is due to the fact that the indirect backscatter term is reduced by the square of the surface reflectivity $\left(R_{v}^{2}=0.17^{2}\right)$. In Fig. 6, the FCF's for both the $V V$ - and $H H$-polarized backscatter responses are shown, which clearly demonstrate the dependence of FCF on polarization. The higher value of $R(\Delta f)$ for $H H$-polarization when compared to $V V$-polarization at $\Delta f=200 \mathrm{MHz}$ is due to the fact that the bistatic scattering phase function $\left(W^{b}\right)$ for $H H$-polarization is twice as high as that for $V V$-polarization. The dependence of the $V V$-polarized FCF on the number density of scatterers for this medium with $d=1 \mathrm{~m}$ and 


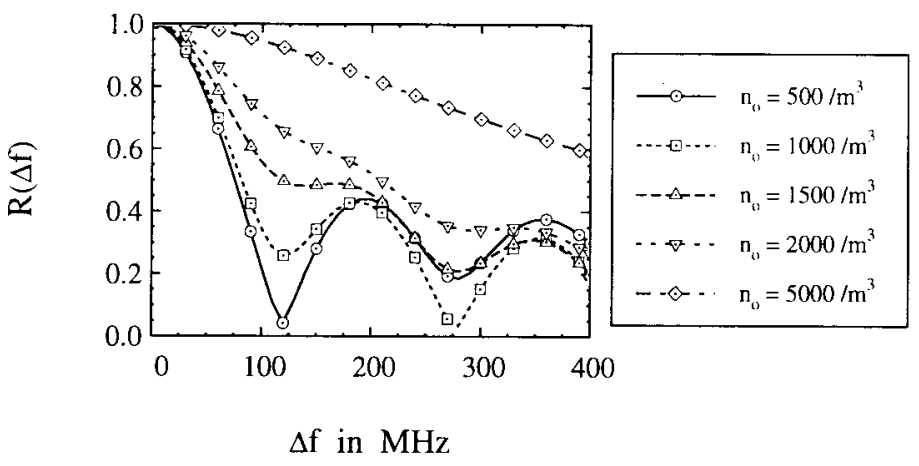

Fig. 7. Dependence of FCF of a tenuous random medium on number density $n_{o}\left(d=1 \mathrm{~m}, \theta_{o}=20^{\circ}, V V\right.$-polarization $)$.

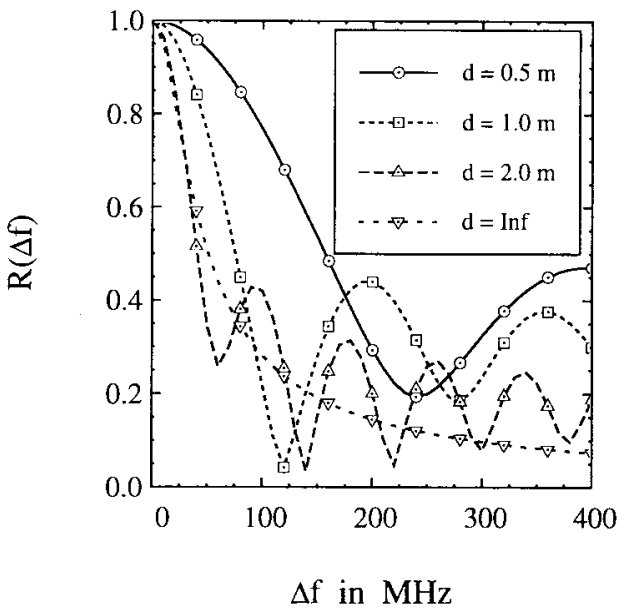

Fig. 8. Dependence of FCF of a tenuous random medium on layer thickness $d\left(n_{o}=500 / \mathrm{m}^{3}, \theta_{o}=20^{\circ}, V V\right.$-polarization).

$\theta=20^{\circ}$ is shown in Fig. 7. It is shown that the backscatter of the medium decorrelates at a slower rate with the shift in frequency as the number density of particles $\left(n_{o}\right)$ increases. This behavior can be explained by noting that the extinction rate in a tenuous medium is directly proportional to $n_{O}$ and the fact that, as $\kappa$ is increased, the bistatic contribution experiences attenuation at a much higher rate than the direct backscatter contribution. That is, the FCF of the medium approaches the FCF of a half-space medium.

The FCF was computed once again, however, this time with $n_{O}$ being fixed at $500 / \mathrm{m}^{3}$ while varying the layer thickness $d$ between $0.5 \mathrm{~m}$ and $\infty$. Fig. 8 demonstrates the dependence of FCF on layer thickness. It is observed that, as the layer thickness is increased, the backscatter decorrelates with the frequency shift at a higher rate (lower correlation bandwidth). Also, more side lobes are generated but with lower amplitudes. This can be attributed to the fact that an increase in the layer thickness results in a decrease in the bistatic term contribution due to an increase in total attenuation $(\kappa d / \cos \theta)$.

Fig. 9 demonstrates the sensitivity of the $V V$-polarized FCF to variations in the incidence angle. In this figure, the layer thickness and number density were kept at $1 \mathrm{~m}$ and $500 / \mathrm{m}^{3}$, respectively. It is observed that the correlation bandwidth increases with the increase in the incidence angle. This can be attributed to two facts: 1) the reflectivity of the lower

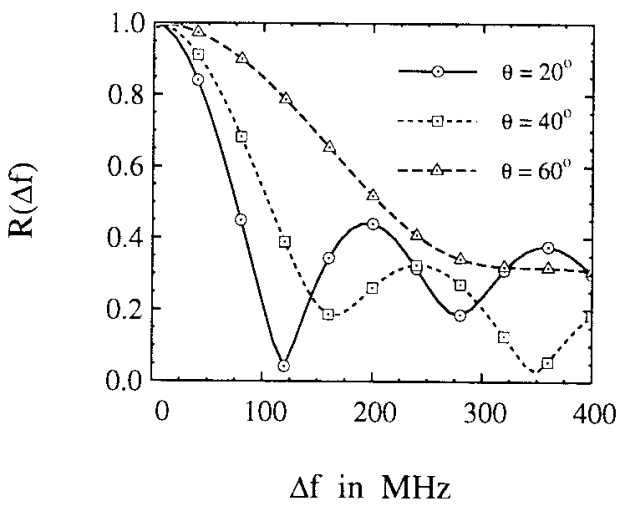

Fig. 9. Dependence of FCF of a tenuous random medium on incidence angle $\theta_{o}\left(d=1 \mathrm{~m}, n_{o}=500 / \mathrm{m}^{3}, V V\right.$-polarization $)$

interface is reduced for $V V$-polarization as the incidence angle is increased, which lowers the contribution from the bistatic term and 2) the overall transmissivity through the layer decreases as the incidence angle increases. The effect of the probability distribution function of the scatterers' orientation angle in elevation on the FCF is demonstrated in Fig. 10(a) for three different p.d.f.'s, namely, uniform, erectophile, and planophile. The three p.d.f.'s are shown in Fig. 10(b). It is observed that the orientation distribution function has a considerable effect on the medium's FCF, especially on the side lobes amplitudes. For example, for an erectophile type medium, where the scatterers are more vertically oriented, the bistatic scattering covariance function is higher than that of either planophile or uniform distributions. In this case, the amplitude of the FCF side lobes is expected to be higher than those of the planophile and uniform distributions.

\section{B. Experimental Observations}

In this section, experimental results are presented that demonstrate the applicability of the FCF for radar inversion of the targets' physical and electrical parameters. Wideband polarimetric backscatter radar responses of rough bare soil surfaces, tenuous random media, and dense random media were measured using the University of Michigan scatterometers operating at $\mathrm{C}-, \mathrm{X}-$, and $\mathrm{W}$-band, respectively. In conducting these experiments, the radars were mounted atop of a boom truck to maintain the far-field distance between the radars and the targets. To characterize the system contribution to the measured FCF, the two-dimensional antenna patterns were measured for each radar in an anechoic chamber following the procedure outlined by Sarabandi et al. [13]. The scatterometers are vector network analyzer-based radars operating in a stepped-frequency mode that permit the measurement of the magnitude and phase of the backscattered fields over a discrete number of frequency points within the radar's prespecified bandwidth. Details on the principle of operation of these scatterometers can be found in [17] and [18].

To compute the FCF of the distributed targets $R_{\text {trgt }}(\Delta f)$, first the FCF of the measured backscattered fields $R_{\mathrm{msr}}(\Delta f)$ was computed, then the system FCF $R_{\text {sys }}(\Delta f)$ was computed using (12), and finally $R_{\text {trgt }}(\Delta f)$ was calculated by dividing $R_{\mathrm{msr}}(\Delta f)$ by $R_{\mathrm{sys}}(\Delta f)$ [see (11)]. To estimate the physical 


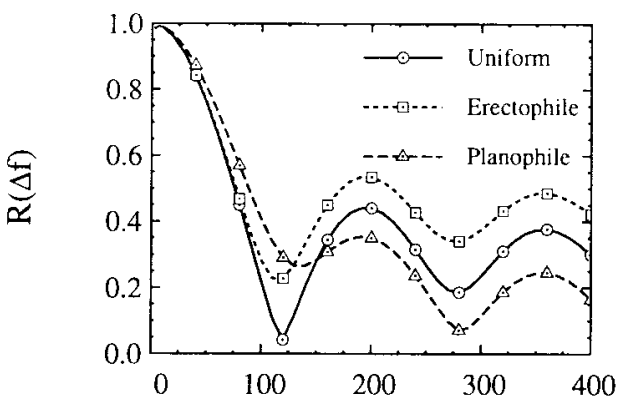

$\Delta \mathrm{f}$ in $\mathrm{MHz}$

(a)

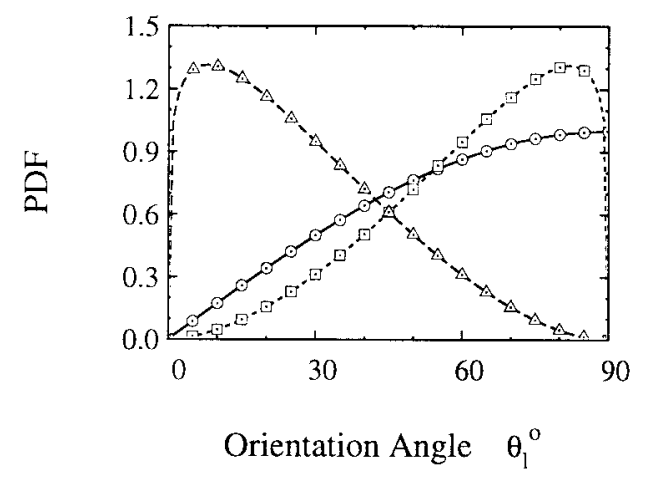

(b)

Fig. 10. (a) Dependence of FCF of a tenuous random medium on the angular distribution function of the particle's orientation angle and (b) different angular distribution functions.

parameters of the distributed target from the measured FCF, a constrained optimization technique [19] was used to minimize the square of the difference between $R_{\text {trgt }}(\Delta f)$ and $R_{\mathrm{mdl}}(\Delta f)$, i.e.,

$$
\min \sum_{n=1}^{N}\left(R_{\text {trgt }}(\Delta f(n))-R_{\text {mdl }}(\Delta f(n))\right)^{2}
$$

with the quantities of interest acting as free parameters. In addition, the optimization routine can be used to minimize the square of the difference between $\Phi_{\mathrm{trgt}}(\Delta f)$ and $\Phi_{\mathrm{mdl}}(\Delta f)$, i.e.,

$$
\min \sum_{n=1}^{N}\left(\Phi_{\text {trgt }}(\Delta f(n))-\Phi_{\text {mdl }}(\Delta f(n))\right)^{2} .
$$

The functions $R_{\mathrm{mdl}}$ and $\Phi_{\mathrm{mdl}}$ in (33) and (34) refer to the theoretical model that characterizes the magnitude and phase of the targets' complex FCF, respectively.

1) Estimation of rms Height of Rough Surfaces: The radar backscatter response of a rough bare soil surface of $4.0-\mathrm{cm}$ rms height and 0.14 volumetric moisture content was measured at an incidence angle of $30^{\circ}$ using our C-band scatterometer. The scatterometer, which was mounted $10.35 \mathrm{~m}$ above the soil surface, is a single antenna system with $5.0^{\circ}$ beamwidth (one way), operating over $400-\mathrm{MHz}$ bandwidth, centered around 5.3 GHz. To achieve a reasonable estimate of FCF of the surface, the polarimetric backscatter responses of more than 100 independent spatial samples were collected. The FCF's

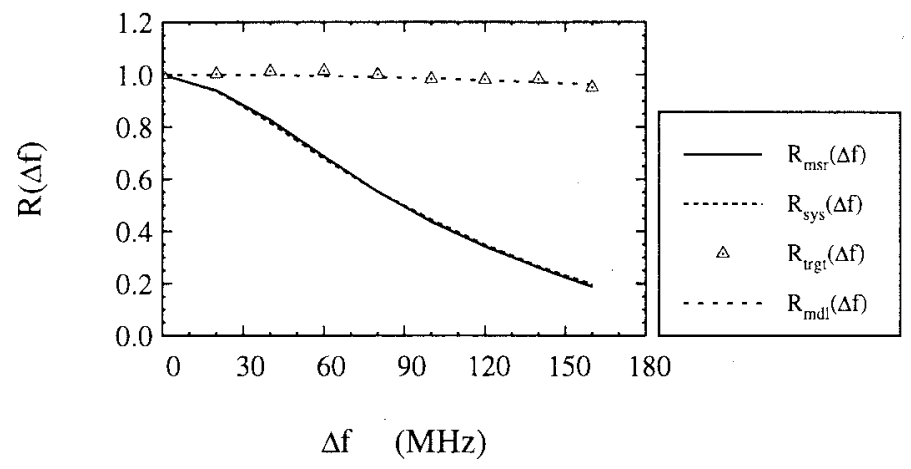

Fig. 11. Comparison between $R_{\mathrm{msr}}^{v v}(\Delta f), R_{\mathrm{sys}}^{v v}(\Delta f), R_{\mathrm{trgt}}^{v v}(\Delta f)$, and $R_{\text {model }}^{v v}(\Delta f)$ of a random rough surface measured at $30^{\circ}$ incidence angle.

$R_{\mathrm{msr}}(\Delta f), R_{\mathrm{sys}}(\Delta f)$, and $R_{\mathrm{trgt}}(\Delta f)$ were computed for the $V V$-polarization configuration and are shown in Fig. 11. It is shown that the measured $R_{\mathrm{msr}}(\Delta f)$ and the computed $R_{\text {sys }}(\Delta f)$ are very similar, implying that the rough surface FCF has a much lower decorrelation rate than the system. To estimate the rms height $s$ of the rough surface, the constrained optimization routine was used to minimize the square of the difference between the measured $R_{\text {trgt }}(\Delta f)$ and $R_{\text {mdl }}(\Delta f)$ computed using (13) with $s$ acting as a free parameter. An rms height of $s=4.7 \mathrm{~cm}$ was obtained, which produces an excellent fit between the measured surface response $R_{\text {trgt }}(\Delta f)$ and the model. The estimated value is in agreement with the measured rms height of the surface.

It should be noted that the total FCF $R_{\mathrm{msr}}$, shown in Fig. 11 is much more sensitive to the system-dependent term $R_{\text {sys }}$ than the surface-dependent term $R_{\text {trgt }}$. Hence, when this systemdependent term is removed, the FCF shows little sensitivity to the surface parameters. To improve the sensitivity, a larger bandwidth and a much gentler $R_{\text {sys }}$ are needed. To reduce the decorrelation rate of the system-dependent term, a radar with a narrow elevation beamwidth operating at near-nadir incidence may be used.

2) Tenuous Random Medium: An X-band scatterometer, mounted $11.3 \mathrm{~m}$ above a grass field, was used to measure the polarimetric backscatter response of more than 100 independent spatial samples of the target at an incidence angle of $20^{\circ}$. The scatterometer operated at a center frequency of 9.5 $\mathrm{GHz}$ with a $500-\mathrm{MHz}$ bandwidth and $6^{\circ}$ antenna beamwidth. The mean grass height and stalk density were measured to be $1.2 \pm 0.3 \mathrm{~m}$ and 80 stalks $/ \mathrm{ft}^{2}$, respectively. The grass biomass and the soil volumetric moisture content were also measured to be $763 \mathrm{~g} / \mathrm{ft}^{2}$ and 0.14 , respectively. The FCF's $R_{\mathrm{msr}}, R_{\mathrm{sys}}$, and $R_{\text {trgt }}$ and their corresponding phase angles $\Phi_{\mathrm{msr}}, \Phi_{\mathrm{sys}}$, and $\Phi_{\text {trgt }}$ [defined in (2)] were computed for the $V V$-polarization configuration and are plotted in Fig. 12(a) and (b). In this case, the optimization routine was used to estimate $\kappa_{v}$ and $Q_{v v}$, and $d$ from the measured magnitude of the complex FCF. As can be seen from Fig. 12(a), an excellent fit was achieved between the measured target response $R_{\text {trgt }}(\Delta f)$ and the model. The estimated values for $\kappa_{v}, Q_{v v}$, and $d$ were found to be $1.04 \mathrm{~Np} / \mathrm{m}, 0.728$, and $1.09 \mathrm{~m}$, respectively. Fig. 12(a) shows the comparison between the model (using the estimated parameters) and the measured $\left(R_{\operatorname{trgt}}(\Delta f)\right)$ FCF's. It is noted 


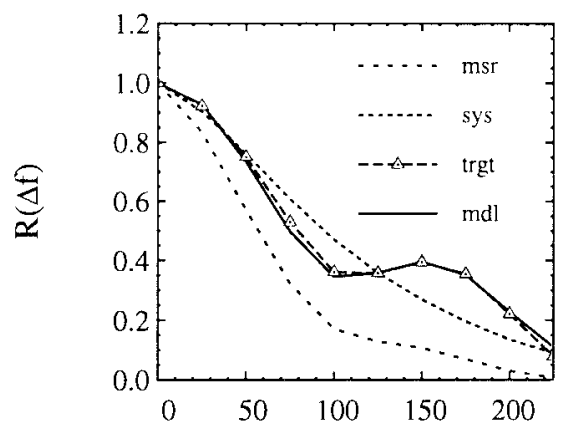

$\Delta \mathrm{f}(\mathrm{MHz})$

(a)

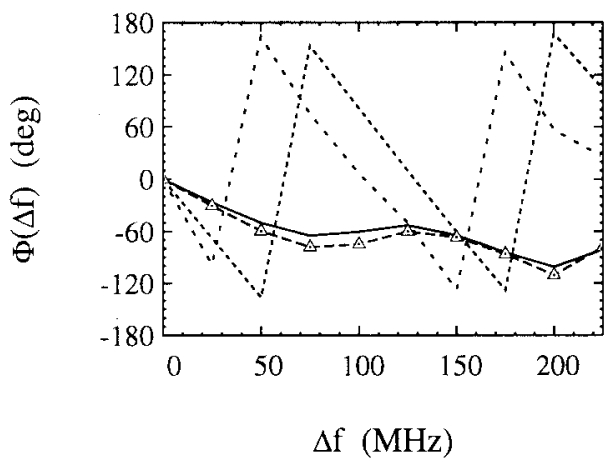

(b)

Fig. 12. Comparison between (a) absolute value and (b) phase angle of the normalized covariance functions $C_{\mathrm{msr}}^{v v}(\Delta f), C_{\mathrm{sys}}^{v v}(\Delta f), C_{\mathrm{trgt}}^{v v}(\Delta f)$, and $C_{\text {model }}^{v v}(\Delta f)$ of a grass field measured at $20^{\circ}$ incidence angle.

here that the estimated depth agreed well with the measured one. As an independent validation tool, the phase angle of $\Phi_{\mathrm{mdl}}(\Delta f)$, which was not used in the minimization process, was computed using $\kappa, Q$, and $d$ and compared to $\Phi_{\text {trgt }}(\Delta f)$. The result is shown in Fig. 12(b), which demonstrates an excellent agreement between $\Phi_{\text {trgt }}(\Delta f)$ and $\Phi_{\text {mdl }}(\Delta f)$. This excellent agreement demonstrates that the estimated parameters were in fact the correct values. Similarly, a good fit was achieved between $R_{\text {trgt }}(\Delta f)$ and $R_{\text {mdl }}(\Delta f)$, for the $H H$-polarization configuration (see Fig. 13). The estimated values for $\kappa_{h}, Q_{h h}$, and $d$ in this case were $0.34 \mathrm{~Np} / \mathrm{m}, 6.13$, $1.05 \mathrm{~m}$, respectively.

3) Estimation of Extinction Rate in Snow: The backscatter response of a thick layer of dry metamorphic snow with fractional volume of 0.34 and mean crystal diameter of 0.5 $\mathrm{mm}$ was measured at $40^{\circ}$ incidence angle using our $\mathrm{W}$ band polarimetric scatterometer. The scatterometer, which was mounted $7.7 \mathrm{~m}$ above the upper snow surface, is a dual antenna system with $1.4^{\circ}$ effective beamwidth operating over 1.0-GHz bandwidth centered around $93.5 \mathrm{GHz}$. During the measurements, the backscatter responses of more than 100 independent spatial samples were collected. The FCF's $R_{\mathrm{msr}}(\Delta f), R_{\mathrm{sys}}(\Delta f)$, and $R_{\mathrm{trgt}}(\Delta f)$, plotted in Fig. 14, were computed for the $V V$-polarization configuration. To estimate the extinction parameter $\kappa$ of the thick layer of snow, the optimization routine was used to estimate $\kappa$ using the semiinfinite FCF expression given by (28). An excellent agreement

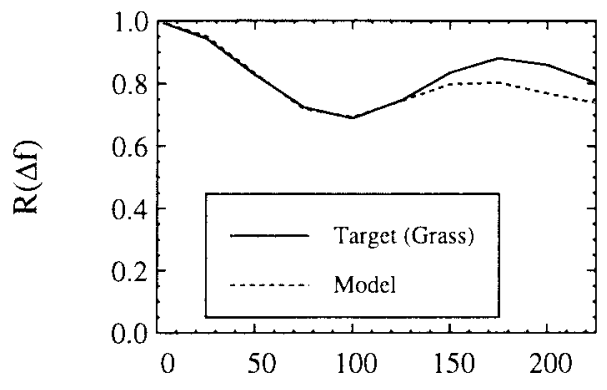

$\Delta \mathrm{f}(\mathrm{MHz})$

Fig. 13. Comparison between the magnitudes of the measured and modeled FCF's of a grass field measured at $20^{\circ}$ incidence angle using the $H H$-polarization configuration.

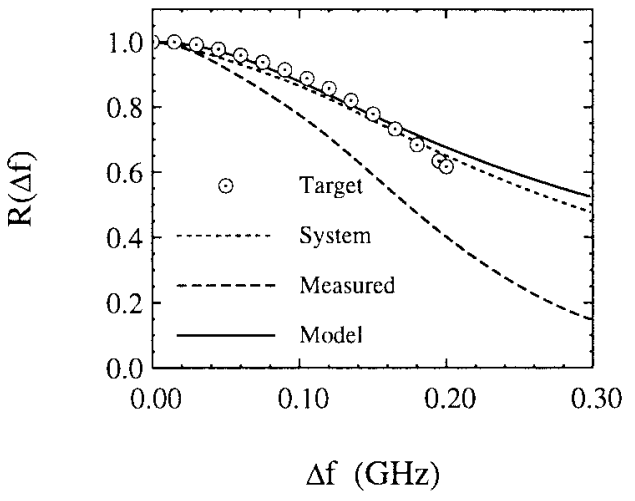

Fig. 14. Comparison between $R_{\mathrm{msr}}^{v v}(\Delta f), R_{\mathrm{sys}}^{v v}(\Delta f), R_{\mathrm{trgt}}^{v v}(\Delta f)$, and $R_{\text {model }}^{v v}(\Delta f)$ of a dry snow field measured at $40^{\circ}$ incidence angle.

between the measured target response $R_{\mathrm{trgt}}(\Delta f)$ and the model is shown in Fig. 14. The estimated value $\kappa_{\text {estim }}(5.0$ $\mathrm{Np} / \mathrm{m})$ falls within the acceptable range of $\kappa(1.9 \mathrm{~Np} / \mathrm{m}<\kappa<$ $30.7 \mathrm{~Np} / \mathrm{m}$ ) for dry snow at W-band frequencies, as reported by Kuga et al. [20]. Furthermore, to verify that the estimated value $\kappa_{\text {estim }}$ is correct, the quasicrystalline approximation with coherent potential technique (QCA-CP) [21] was used to compute the extinction. QCA-CP predicts an extinction rate of $\kappa_{\mathrm{qca}-c p}=6.1 \mathrm{~Np} / \mathrm{m}$ (assuming $\epsilon_{\text {ice }}=3.15+i 0.0085$ ) that agrees well with estimated $\kappa_{\text {estim }}=5 \mathrm{~Np} / \mathrm{m}$ using FCF measurements.

\section{CONCLUSIONS}

The potential of backscatter FCF as a useful remote-sensing tool was demonstrated in this paper. Analytical expressions for the complex FCF of simple random media and a specific class of rough surfaces were derived. The results were obtained for polarimetric radars with arbitrary radiation patterns. It was shown that the FCF of statistically homogeneous distributed targets are functions of both the target and radar attributes and that the expressions for the FCF can be written in terms of the product of two expressions, one only a function of the system parameters and the other only a function of the target parameters. A sensitivity study was carried out to demonstrate the sensitivity of FCF on the electrical and physical parameters of the target. It was found that the behavior of FCF, such as the 
decorrelation bandwidth and the number of side-lobes and their level, are strong functions of target attributes. Experiments were conducted using wideband scatterometers at $\mathrm{C}-, \mathrm{X}-$, and $\mathrm{W}$-band on tenuous and nontenuous random media and rough surfaces to demonstrate the feasibility of target parameter retrieval. For the inversion process, a standard optimization algorithm in conjunction with the theoretical FCF models were used. The success of the inversion procedure was demonstrated by comparing the estimated parameters with the ground truth data and/or other independent methods.

\section{REFERENCES}

[1] D. L. Evans, T. G. Farr, J. P. Ford, T. W. Thompson, and C. L. Werner, "Multipolarization radar images for geologic mapping and vegetation discrimination," IEEE Trans. Geosci. Remote Sensing, vol. GE-24, p. 246, Mar. 1986.

[2] K. Sarabandi, "Electromagnetic scattering from vegetation canopies," Ph.D. dissertation, Univ. of Michigan, Ann Arbor, 1989.

[3] M. C. Dobson, F. T. Ulaby, L. E. Pirece, T. L. Sharik, K. M. Bergen, J. Kellndorfer, J. R. Kendra, E. Li, Y. C. Lin, A. Nashashibi, K. Sarabandi, and P. Siqueira, "Estimation of forest biomass," IEEE Trans. Geosci. Remote Sensing, vol. 33, pp. 887-895, July 1995.

[4] K. Sarabandi and Y.-C. Lin, "Simulation of interferometric SAR response for characterization of scattering phase center statistics of forest canopies," IEEE Trans. Geosci. Remote Sensing, to be published.

[5] J. T. Ransone and J. W. Wright, "A radar ocean wave spectrometers," in Proc. IEEE Intl. Conf. Eng. Ocean Environ., Newport, RI, Sept. 1972.

[6] D. E. Weissman, "Two frequency radar interferometry applied to the measurement of ocean wave height," IEEE Trans. Antennas Propagat., vol. AP-21, pp. 649-656, Sept. 1973

[7] D. E. Weissman and J. W. Johnson, "Dual frequency correlation radar measurements of the height statistics of ocean waves," IEEE Trans. Antenna Propagat., vol. AP-25, pp. 74-83, Jan. 1977.

[8] A. A. Monakov, J. Vivekanandan, A. S. Stjernman, and A. K. Nystrom, "Spatial and frequency averaging techniques for a polarimetric scatterometer system," IEEE Trans. Geosci. Remote Sensing, vol. 32, pp. 187-196, Jan. 1994

[9] F. T. Ulaby, T. F. Haddock, and R. T. Austin, "Fluctuation statistics of millimeter-wave scattering from distributed targets," IEEE Trans. Geosci. Remote Sensing, vol. 26, pp. 268-281, May 1988.

[10] T. Chan, Y. Kuga, and A. Ishimaru, "Subsurface detection of a buried object using angular correlation function measurement," Wave Random Media, vol. 7, pp. 457-465, 1997.

[11] K. Sarabandi, " $\Delta k$-radar equivalent of interferometric SAR's: A theoretical study for determination of vegetation height," IEEE Trans. Geosci. Remote Sensing, vol. 35, pp. 1267-1276, Sept. 1997.
[12] Y. Oh, K. Sarabandi, and F. T. Ulaby, "An empirical and an inversion technique for radar scattering from bare soil surfaces," IEEE Trans. Geosci. Remote Sensing, vol. 30, pp. 370-381, Mar. 1992.

[13] K. Sarabandi, Y. Oh, and F. T. Ulaby, "Measurement and calibration of differential Mueller matrix of distributed targets," IEEE Trans. Antenna Propagat., vol. 40, pp. 1524-1532, Dec. 1992.

[14] F. T. Ulaby, R. K. Moore, and A. K. Fung, Microwave Remote Sensing, vol II. Norwell, MA: Artech House, 1986.

[15] L. Tsang, J. A. Kong, and R. T. Shin, Theory of Microwave Remote Sensing. New York: Wiley, 1985.

[16] T. B. A. Senior, K. Sarabandi, and F. T. Ulaby, "Measuring and modeling the backscattering cross section of a leaf," Radio Sci., vol. 22, pp. 1109-1116, Nov. 1987.

[17] F. T. Ulaby and C. Elachi, Radar Polarimetry for Geoscience Applications. Norwell, MA: Artech House, 1990

[18] F. T. Ulaby, M. Whitt, and K. Sarabandi, "AVNA-based polarimetric scatterometers," IEEE AP Mag., vol. 32, 1990.

[19] J. L. Zhou and A. L. Tits, "User's guide for FSQP version 3.3b: A FORTRAN code for solving constrained nonlinear (minimax) optimization problems, generating iterates satisfying all inequality and linear contraints,” Elect. Eng. Depart. Inst. Syst. Res., Univ. of Maryland, College Park.

[20] Y. Kuga, F. T. Ulaby, T. F. Haddock, and R. D. DeRoo, "Millimeterwave radar scattering from snow 1. Radiative transfer model," Radio Sci., vol. 26, pp. 329-341, Mar.-Apr. 1991.

[21] B. Wen, L. Tsang, D. P. Winebrenner, and A. Ishimaru, "Dense medium radiative transfer theory: Comparison with experiment and application to microwave remote sensing and polarimetry," IEEE Trans. Geosci. Remote Sensing, vol. 28, pp. 46-59, Jan. 1990.

Kamal Sarabandi (S'87-M'90-SM'92) for a photograph and biography, see p. 35 of the January 1999 issue of this TRANSACTIONS.

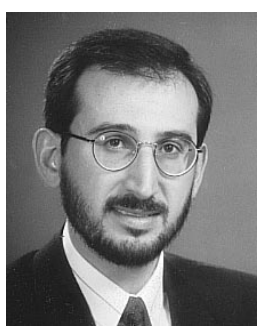

Adib Nashashibi (S'82-M'95) received the B.Sc. and M.Sc. degrees in electrical engineering from Kuwait University, Kuwait, in 1985 and 1988, respectively. He received the Ph.D. degree in electrical engineering from the University of Michigan, Ann Arbor, in 1995.

$\mathrm{He}$ is an Assistant Research Scientist in the Radiation Laboratory, The University of Michigan. His research interests include microwave remotesensing, polarimetric millimeter-wave radars, calibration and measurement techniques, electromagnetic wave propagation, and scattering in random media. 